\title{
EXISTENCE THEORY FOR CERTAIN ORDINARY DIFFERENTIAL EQUATIONS WITH A MONOTONE SINGULARITY ${ }^{1}$
}

\author{
KEITH MILI,ER
}

The following result arose in the course of some work [2] on extremal solutions of uniformly elliptic partial differential equations. All variables and functions here are real valued and $n$ is a positive integer.

Theorem. Suppose $G\left(t, x_{0}, \cdots, x_{n-1}\right)$ is a function which is $(n+1)$ dimensionally continuous for $t \geqq 0,-\infty<x_{0}<\infty, \cdots,-\infty<x_{n-1}$ $<\infty$, uniformly Lipschitz in $x_{0}, \cdots, x_{n-1}$ for $t \geqq 0$, and which is monotone nonincreasing with respect to $x_{n-1}$ for $t$ in some neighborhood of 0 . Let $p_{0}, \cdots, p_{n-2}$ be continuous functions of $t$ which are bounded outside every neighborhood of 0 , and such that $p_{i}(t)=o\left(t^{(i-n)}\right)$ as $t \rightarrow 0, i=0$, $\cdots, n-2$. There then exists a unique solution of the initial value problem

$$
\begin{aligned}
f & \in C^{n}, \quad t \geqq 0, \\
f^{(n)}(t) & =G\left(t, p_{0} f^{(0)}, \cdots, p_{n-2} f^{(n-2)}, t^{-1} f^{(n-1)}\right), \quad t>0, \\
f^{(i)}(0) & =0, \quad i=0, \cdots, n-1 .
\end{aligned}
$$

We point out that it suffices to show existence and uniqueness for (1) in some neighborhood $[0, \delta]$ of the origin, $\delta>0$. Since $G$ is uniformly Lipschitz in $f^{(0)}, \cdots, f^{(n-1)}$ for $t \geqq \delta$, the solution could be uniquely continued from $t=\delta$ using the usual Picard existence and uniqueness theory [1]. The proof now proceeds by way of several lemmas.

Lemma 1. If $f$ is a solution of (1) then $f_{n} \equiv f^{(n)}(0)$ must be the unique fixed point of the equation

$$
f_{n}=G\left(0, \cdots, 0, f_{n}\right) .
$$

Proof. If $f$ is $C^{n}$ up to the origin then

$$
\begin{aligned}
t^{-1} f^{(n-1)}(t) & =f_{n}+o(1), \\
p_{i}(t) f^{(i)}(t) & =o(1), \quad i=0, \cdots, n-2,
\end{aligned}
$$

Received by the editors November 18, 1966.

1 This work was supported by the Air Force Office of Scientific Research and the National Academy of Sciences through a Postdoctoral Research Fellowship for 19641965 at the University of Genova and also by AFOSR grant number 553-64. 
as $t \rightarrow 0$. Hence, taking limits in (1) we obtain (2). The fixed point of (2) exists and is unique since $G$ is continuous and nonincreasing in the last argument.

Lemma 2. Given $\epsilon>0$ there exists an "€-approximate solution" $f_{\epsilon}$ satisfying

$$
\begin{array}{rlrl}
f_{\epsilon} & \in C^{n}, & t \geqq 0, \\
\left|f_{\epsilon}^{(n)}(t)-G\left(t, p_{0} f_{\epsilon}^{(0)}, \cdots, t^{-1} f_{\epsilon}^{(n-1)}\right)\right| \leqq \epsilon, & t>0, \\
f_{\epsilon}^{(i)}(0) & =0, & i=0, \cdots, n-1 .
\end{array}
$$

Proof. Let $f_{\epsilon}=t^{n} f_{n} / n$ ! for $0 \leqq t \leqq \eta$. Then

$$
\epsilon(t) \equiv f_{\epsilon}^{(n)}(t)-G\left(t, p_{0} f_{\epsilon}^{(0)}, \cdots, t^{-1} f_{\epsilon}^{(n-1)}\right)
$$

is a continuous function which $\rightarrow 0$ as $t \rightarrow 0$. Choose $\eta$ sufficiently small that $|\boldsymbol{\epsilon}(t)|<\epsilon$ for $0 \leqq t \leqq \eta$. Extend $\boldsymbol{\epsilon}(t)$ continuously to zero in some neighborhood past $t=\eta$, always keeping $|\epsilon(t)|<\epsilon$ globally. Then, for $t>\eta$, let $f_{\epsilon}$ be defined to be a solution of

$$
\begin{aligned}
& f_{\epsilon}^{(n)}(t)=G\left(t, p_{0} f^{(0)}, \cdots, t^{-1} f^{(n-1)}\right)+\epsilon(t), \quad t>\eta, \\
& f_{\epsilon}^{(i)}(\eta)=\eta^{(n-i)} f_{n} /(n-i) !, \quad i=0, \cdots, n-1 .
\end{aligned}
$$

Such a $C^{n}$ solution exists by the usual Cauchy-Peano theory [1]. Clearly $f_{\epsilon}$ patches together in a $C^{n}$ fashion at $t=\eta$, thus giving a solution of (4) as desired.

Lemma 3. Let $f$ and $g$ be two solutions of (4). Let $K_{i}$ be the Lipschitz constant of $G$ with respect to $X_{i}, i=0, \cdots, n-2$. Let $\delta>0$ be sufficiently small that $K_{\delta}=\sup _{t \in[0, \delta]} \sum_{i=0}^{n-2} K_{i} p^{i}(t) t^{(n-i)} /(n-i)$ ! is less than 1 and that $[0, \delta]$ lies within the interval of monotonicity of $G$ with respect to its last argument. Then

$$
\begin{aligned}
\left|(f-g)^{(i)}(t)\right| \leqq\left(2 \epsilon /\left(1-K_{\delta}\right)\right) t^{(n-i)} /(n-i) !, & \\
& i=0, \cdots, n-1, \quad 0 \leqq t \leqq \delta .
\end{aligned}
$$

Proof. Let $y=f-g$. Now $\left|y^{(n-1)}\right|^{\prime}=y^{(n)}$ when $y^{(n-1)}>0$ and $\left|y^{(n-1)}\right|^{\prime}=-y^{(n)}$ when $y^{(n-1)}<0$. Thus, subtracting equations for $f$ and $g$ and using the monotonicity of $G$, one sees that either $\left|y^{(n-1)}\right|=0$, or

$$
\left|y^{(n-1)}\right|^{\prime} \leqq K_{0}\left|p_{0} y^{(0)}\right|+\cdots K_{n-2}\left|p_{n-2} y^{(n-2)}\right|+2 \epsilon .
$$

Let $[0, \delta]^{*}$ be the subset of $[0, \delta]$ where $\left|y^{(n-1)}\right| \neq 0$ and let 


$$
m=\sup _{\tau \in[0, \delta]^{*}}\left|y^{(n-1)}\right|^{\prime}(\tau) .
$$

One easily shows that

$$
\left|y^{(i)}(t)\right| \leqq m t^{(n-i)} /(n-i) !, \quad i=0, \cdots, n-1,0 \leqq t \leqq \delta .
$$

Inserting this for $i=0, \cdots, n-2$ into (8) and taking supremums over $[0, \delta]^{*}$ one has $m \leqq 2 \epsilon /\left(1-K_{\delta}\right)$ as desired.

Proof of the Theorem. Let $\epsilon_{j} \rightarrow 0$ as $j \rightarrow \infty$. Then, by the a priori bounds (7), $p_{0} f_{\epsilon_{j}}^{(0)}, \cdots, t^{-1} f_{\epsilon_{j}}^{(n-1)}$ are all uniformly Cauchy convergent on $[0, \delta]$. Thus, by (4) and the continuity of $G$ in all its variables, $f_{\epsilon_{j}}^{(n)}$ is also uniformly Cauchy convergent on $[0, \delta]$. The limit function is therefore a $C^{n}$ solution of (1) on $[0, \delta]$ and the proof is completed. Note. The local existence and uniqueness on $[0, \delta], \delta$ defined as in Lemma 3, in no way utilizes the Lipschitz condition on $G$ with respect to its last argument. This condition was required only for the global uniqueness.

\section{REFERENCES}

1. E. Coddington and N. Levinson, Theory of ordinary differential equations, McGraw-Hill, New York, 1955.

2. K. Miller, Extremal barriers on cones with Phragmen-Lindelöf theorems and other applications, (to appear).

University of California, Berkeley 